FACTA UNIVERSITATIS (NIŠ)

Ser. Math. Inform. Vol. 33, No 2 (2018), 269-277

https://doi.org/10.22190/FUMI1802269B

\title{
REMARKS ON METALLIC WARPED PRODUCT MANIFOLDS
}

\author{
Adara M. Blaga and Cristina E. Hreţcanu
}

\begin{abstract}
We characterize the metallic structure on the product of two metallic manifolds in terms of metallic maps and provide a necessary and sufficient condition for the warped product of two locally metallic Riemannian manifolds to be locally metallic. We discuss a particular case of the product manifolds and we construct an example of the metallic warped product Riemannian manifold.

Keywords: Riemannian manifold, metallic warped product, projection mapping.
\end{abstract}

\section{Introduction}

Starting from a polynomial structure, which was generally defined by S. I. Goldberg, K. Yano and N. C. Petridis in $([8],[9])$, we consider a polynomial structure on an $m$-dimensional Riemannian manifold $(M, g)$, called by us a metallic structure $([6],[11],[7],[12])$, determined by a $(1,1)$-tensor field $J$ which satisfies the equation:

$$
J^{2}=p J+q I
$$

where $I$ is the identity operator on the Lie algebra of vector fields on $M$ identified with the set of smooth sections $\Gamma(T(M)$ ) (and we will simply denote $X \in T(M)$ ) with $p$ and $q$ are non-zero natural numbers). From the definition, we easily get the recurrence relation:

$$
J^{n+1}=g_{n+1} \cdot J+g_{n} \cdot I,
$$

where $\left(\left\{g_{n}\right\}_{n \in \mathbb{N}^{*}}\right)$ is the generalized secondary Fibonacci sequence defined by $g_{n+1}=$ $p g_{n}+q g_{n-1}, n \geq 1$ with $g_{0}=0, g_{1}=1$ and $p, q \in \mathbb{N}^{*}$.

If $(M, g)$ is a Riemannian manifold endowed with a metallic structure $J$ such that the Riemannian metric $g$ is $J$-compatible (i.e. $g(J X, Y)=g(X, J Y)$, for any $X, Y \in T(M))$, then $(M, g, J)$ is called a metallic Riemannian manifold. In this case:

$$
\text { (1.3) } \quad g(J X, J Y)=p g(X, J Y)+q g(X, Y),
$$

Received March 30, 2018; accepted May 24, 2018

2010 Mathematics Subject Classification. Primary 53C15; Secondary 53C25 
for any $X, Y \in T(M)$.

It is known ([13]) that an almost product structure $F$ on $M$ induces two metallic structures:

$$
J_{ \pm}= \pm \frac{2 \sigma_{p, q}-p}{2} F+\frac{p}{2} I
$$

and, conversely, every metallic structure $J$ on $M$ induces two almost product structures:

$$
F_{ \pm}= \pm \frac{2}{2 \sigma_{p, q}-p} J-\frac{p}{2 \sigma_{p, q}-p} I
$$

where $\sigma_{p, q}=\frac{p+\sqrt{p^{2}+4 q}}{2}$ is the metallic number, which is a positive solution of the equation $x^{2}-p x-q=0$, for $p$ and $q$ non-zero natural numbers.

In particular, if the almost product structure $F$ is compatible with the Riemannian metric, then $J_{+}$and $J_{-}$are metallic Riemannian structures.

On a metallic manifold $(M, J)$ there exist two complementary distributions $\mathcal{D}_{l}$ and $\mathcal{D}_{m}$ corresponding to the projection operators $l$ and $m([13])$ given by:

$$
l=-\frac{1}{2 \sigma_{p, q}-p} J+\frac{\sigma_{p, q}}{2 \sigma_{p, q}-p} I, \quad m=\frac{1}{2 \sigma_{p, q}-p} J+\frac{\sigma_{p, q}-p}{2 \sigma_{p, q}-p} I .
$$

The analogue concept of a locally product manifold is considered in the context of metallic geometry. Precisely, we say that the metallic Riemannian manifold $(M, g, J)$ is locally metallic if $J$ is parallel with respect to the Levi-Civita connection associated to $g$.

\section{Metallic warped product Riemannian manifolds}

\subsection{Warped product manifolds}

Let $\left(M_{1}, g_{1}\right)$ and $\left(M_{2}, g_{2}\right)$ be two Riemannian manifolds of dimensions $n$ and $m$, respectively. Denote by $p_{1}$ and $p_{2}$ the projection maps from the product manifold $M_{1} \times M_{2}$ onto $M_{1}$ and $M_{2}$ and by $\widetilde{\varphi}:=\varphi \circ p_{1}$ the lift to $M_{1} \times M_{2}$ of a smooth function $\varphi$ on $M_{1}$. In this case, we call $M_{1}$ the base and $M_{2}$ the fiber of $M_{1} \times M_{2}$. The unique element $\tilde{X}$ of $T\left(M_{1} \times M_{2}\right)$ that is $p_{1}$-related to $X \in T\left(M_{1}\right)$ and to the zero vector field on $M_{2}$ will be called the horizontal lift of $X$ and the unique element $\widetilde{V}$ of $T\left(M_{1} \times M_{2}\right)$ that is $p_{2}$-related to $V \in T\left(M_{2}\right)$ and to the zero vector field on $M_{1}$ will be called the vertical lift of $V$. Also denote by $\mathcal{L}\left(M_{1}\right)$ the set of all horizontal lifts of vector fields on $M_{1}$ and by $\mathcal{L}\left(M_{2}\right)$ the set of all vertical lifts of vector fields on $M_{2}$.

For $f>0$ a smooth function on $M_{1}$, consider the Riemannian metric on $M_{1} \times M_{2}$ :

$$
\widetilde{g}:=p_{1}^{*} g_{1}+\left(f \circ p_{1}\right)^{2} p_{2}^{*} g_{2} .
$$

Definition 2.1. ([4]) The product manifold of $M_{1}$ and $M_{2}$ together with the Riemannian metric $\widetilde{g}$ defined by (2.1) is called the warped product of $M_{1}$ and $M_{2}$ by the warping function $f$ [and it is denoted by $\left.\left(\widetilde{M}:=M_{1} \times_{f} M_{2}, \widetilde{g}\right)\right]$. 
Note that if $f$ is constant (equal to 1 ), the warped product becomes the usual product of the Riemannian manifolds.

For $(x, y) \in \widetilde{M}$, we shall identify $X \in T\left(M_{1}\right)$ with $\left(X_{x}, 0_{y}\right) \in T_{(x, y)}(\widetilde{M})$ and $Y \in T\left(M_{2}\right)$ with $\left(0_{x}, Y_{y}\right) \in T_{(x, y)}(\widetilde{M})([3])$.

The projection mappings of $T\left(M_{1} \times M_{2}\right)$ onto $T\left(M_{1}\right)$ and $T\left(M_{2}\right)$, respectively, denoted by $\pi_{1}=: T p_{1}$ and $\pi_{2}=: T p_{2}$ verify:

$$
\pi_{1}+\pi_{2}=I, \quad \pi_{1}^{2}=\pi_{1}, \quad \pi_{2}^{2}=\pi_{2}, \quad \pi_{1} \circ \pi_{2}=\pi_{2} \circ \pi_{1}=0 .
$$

The Riemannian metric of the warped product manifold $\widetilde{M}=M_{1} \times_{f} M_{2}$ equals to:

$$
\widetilde{g}(\widetilde{X}, \widetilde{Y})=g_{1}\left(X_{1}, Y_{1}\right)+\left(f \circ p_{1}\right)^{2} g_{2}\left(X_{2}, Y_{2}\right)
$$

for any $\widetilde{X}=\left(X_{1}, X_{2}\right), \widetilde{Y}=\left(Y_{1}, Y_{2}\right) \in T(\widetilde{M})=T\left(M_{1} \times_{f} M_{2}\right)$ and we notice that the leaves $M_{1} \times\{y\}$, for $y \in M_{2}$, are totally geodesic submanifolds of $\left(\widetilde{M}=M_{1} \times_{f} M_{2}, \widetilde{g}\right)$.

If we denote by $\widetilde{\nabla},{ }^{M_{1}} \nabla,{ }^{M_{2}} \nabla$ the Levi-Civita connections on $\widetilde{M}, M_{1}$ and $M_{2}$, we know that for any $X_{1}, Y_{1} \in T\left(M_{1}\right)$ and $X_{2}, Y_{2} \in T\left(M_{2}\right)$ ([14]):

$$
\begin{gathered}
\widetilde{\nabla}_{\left(X_{1}, X_{2}\right)}\left(Y_{1}, Y_{2}\right)=\left({ }^{M_{1}} \nabla_{X_{1}} Y_{1}-\frac{1}{2} g_{2}\left(X_{2}, Y_{2}\right) \cdot \operatorname{grad}\left(f^{2}\right)\right. \\
\left.{ }^{M_{2}} \nabla_{X_{2}} Y_{2}+\frac{1}{2 f^{2}} X_{1}\left(f^{2}\right) Y_{2}+\frac{1}{2 f^{2}} Y_{1}\left(f^{2}\right) X_{2}\right)
\end{gathered}
$$

In particular:

$$
\widetilde{\nabla}_{(X, 0)}(0, Y)=\widetilde{\nabla}_{(0, Y)}(X, 0)=(0, X(\ln (f)) Y)
$$

Let $R, R_{M_{1}}, R_{M_{2}}$ be the Riemannian curvature tensors on $\widetilde{M}, M_{1}$ and $M_{2}$ and $\widetilde{R_{M_{1}}}, \widetilde{R_{M_{2}}}$ the lift on $\widetilde{M}$ of $R_{M_{1}}$ and $R_{M_{2}}$. Then:

Lemma 2.1. ([4]) If $\left(\widetilde{M}:=M_{1} \times_{f} M_{2}, \widetilde{g}\right)$ is the warped product of $M_{1}$ and $M_{2}$ by the warping function $f$ and $m>1$, then for any $X, Y, Z \in \mathcal{L}\left(M_{1}\right)$ and any $U$, $V, W \in \mathcal{L}\left(M_{2}\right)$, we have:

1. $R(X, Y) Z=\widetilde{R_{M_{1}}}(X, Y) Z$;

2. $R(U, X) Y=\frac{1}{f} H^{f}(X, Y) U$, where $H^{f}$ is the lift on $\widetilde{M}$ of $H \operatorname{ess}(f)$;

3. $R(X, Y) U=R(U, V) X=0$;

4. $R(U, V) W=\widetilde{R_{M_{2}}}(U, V) W-\frac{|\operatorname{grad}(f)|^{2}}{f^{2}}[g(U, W) V-g(V, W) U]$;

5. $R(X, U) V=\frac{1}{f} g(U, V) \widetilde{\nabla}_{X} \operatorname{grad}(f)$. 
Let $S, S_{M_{1}}, S_{M_{2}}$ be the Ricci curvature tensors on $\widetilde{M}, M_{1}$ and $M_{2}$ and $\widetilde{S_{M_{1}}}$, $\widetilde{S_{M_{2}}}$ the lift on $\widetilde{M}$ of $S_{M_{1}}$ and $S_{M_{2}}$. Then:

Lemma 2.2. ([4]) If $\left(\widetilde{M}:=M_{1} \times_{f} M_{2}, \widetilde{g}\right)$ is the warped product of $M_{1}$ and $M_{2}$ by the warping function $f$ and $m>1$, then for any $X, Y \in \mathcal{L}\left(M_{1}\right)$ and any $V$, $W \in \mathcal{L}\left(M_{2}\right)$, we have:

1. $S(X, Y)=\widetilde{S_{M_{1}}}(X, Y)-\frac{m}{f} H^{f}(X, Y)$, where $H^{f}$ is the lift on $\widetilde{M}$ of $H e s s(f)$;

2. $S(X, V)=0$;

3. $S(V, W)=\widetilde{S_{M_{2}}}(V, W)-\left[\frac{\Delta(f)}{f}+(m-1) \frac{|\operatorname{grad}(f)|^{2}}{f^{2}}\right] g(V, W)$.

Remark 2.1. For the case of product Riemannian manifolds:

i) the Riemannian curvature tensors verify ([2]):

$$
R(\tilde{X}, \widetilde{Y}) \widetilde{Z}=\left(R_{1}\left(X_{1}, Y_{1}\right) Z_{1}, R_{2}\left(X_{2}, Y_{2}\right) Z_{2}\right),
$$

for any $\widetilde{X}=\left(X_{1}, X_{2}\right), \widetilde{Y}=\left(Y_{1}, Y_{2}\right), \widetilde{Z}=\left(Z_{1}, Z_{2}\right) \in T\left(M_{1} \times M_{2}\right)$, where $R, R_{1}$ and $R_{2}$ are respectively the Riemannian curvature tensors of the Riemannian manifolds $\left(M_{1} \times M_{2}, \widetilde{g}\right)$, $\left(M_{1}, g_{1}\right)$ and $\left(M_{2}, g_{2}\right)$;

ii) the Ricci curvature tensors verify ([2]):

$$
S(\widetilde{X}, \widetilde{Y})=S_{1}\left(X_{1}, Y_{1}\right)+S_{2}\left(X_{2}, Y_{2}\right),
$$

for any $\tilde{X}=\left(X_{1}, X_{2}\right), \widetilde{Y}=\left(Y_{1}, Y_{2}\right) \in T\left(M_{1} \times M_{2}\right)$, where $S, S_{1}$ and $S_{2}$ are respectively the Ricci curvature tensors of the Riemannian manifolds $\left(M_{1} \times M_{2}, \widetilde{g}\right),\left(M_{1}, g_{1}\right)$ and $\left(M_{2}, g_{2}\right)$.

Note that the Riemannian curvature tensor of a locally metallic Riemannian manifold has the following properties:

Proposition 2.1. If $(M, g, J)$ is a locally metallic Riemannian manifold, then for any $X, Y, Z \in T(M)$ :

$$
\begin{gathered}
R(X, Y) J Z=J(R(X, Y) Z), \\
R(J X, Y)=R(X, J Y), \\
R(J X, J Y)=q R(J X, Y)+p R(X, Y), \\
R\left(J^{n+1} X, Y\right)=g_{n+1} \cdot R(J X, Y)+g_{n} \cdot R(X, Y),
\end{gathered}
$$

where $\left(\left\{g_{n}\right\}_{n \in \mathbb{N}^{*}}\right)$ is the generalized secondary Fibonacci sequence defined by $g_{n+1}=$ $p g_{n}+q g_{n-1}, n \geq 1$ with $g_{0}=0, g_{1}=1$ and $p, q \in \mathbb{N}^{*}$.

Proof. The locally metallic condition $\nabla J=0$ is equivalent to $\nabla_{X} J Y=J\left(\nabla_{X} Y\right)$, for any $X, Y \in T(M)$ and (2.7) follows from the definition of $R$. The relations (2.8), (2.9) and (2.10) follow from the symmetries of $R$ and from the recurrence relation $J^{n+1}=g_{n+1} \cdot J+g_{n} \cdot I$. 
Theorem 2.1. If $\left(\widetilde{M}:=M_{1} \times_{f} M_{2}, \widetilde{g}, \widetilde{J}\right)$ is a locally metallic Riemannian warped product manifold, then $M_{2}$ is $\widetilde{J}$-invariant submanifold of $\widetilde{M}$.

Proof. Applying (2.8) from Proposition 2.1 and Lemma 2.1, we obtain $H^{f}(X, Y) \widetilde{J} U=$ $H^{f}(\widetilde{J} X, Y) U$, for any $X, Y \in \mathcal{L}\left(M_{1}\right)$ and any $U \in \mathcal{L}\left(M_{2}\right)$, where $H^{f}$ is the lift on $\widetilde{M}$ of $\operatorname{Hess}(f)$.

\subsection{Metallic warped product Riemannian manifolds}

\subsubsection{Metallic Riemannian structure on $(\widetilde{M}, \widetilde{g})$ induced by the projection operators}

The endomorphism

$$
F:=\pi_{1}-\pi_{2}
$$

verifies $F^{2}=I$ and $\widetilde{g}(F \widetilde{X}, \widetilde{Y})=\widetilde{g}(\widetilde{X}, F \widetilde{Y})$, thus $F$ is an almost product structure on $M_{1} \times M_{2}$.

By using relations (1.4) we can construct on $M_{1} \times M_{2}$ two metallic structures, given by:

$$
\widetilde{J}_{ \pm}= \pm \frac{2 \sigma_{p, q}-p}{2} F+\frac{p}{2} I
$$

Also from $\widetilde{g}(F \tilde{X}, \widetilde{Y})=\widetilde{g}(\widetilde{X}, F \widetilde{Y})$ follows $\widetilde{g}\left(\widetilde{J_{ \pm}} \widetilde{X}, \widetilde{Y}\right)=\widetilde{g}\left(\widetilde{X}, \widetilde{J}_{ \pm} \widetilde{Y}\right)$. Therefore, we can state the following result:

Theorem 2.2. There exist two metallic Riemannian structures $\widetilde{J}_{ \pm}$on $(\widetilde{M}, \widetilde{g})$ given by:

$$
\widetilde{J}_{ \pm}= \pm \frac{2 \sigma_{p, q}-p}{2} F+\frac{p}{2} I
$$

where $\widetilde{M}=M_{1} \times_{f} M_{2}$ and $\widetilde{g}(\widetilde{X}, \widetilde{Y})=g_{1}\left(X_{1}, Y_{1}\right)+\left(f \circ p_{1}\right)^{2} g_{2}\left(X_{2}, Y_{2}\right)$, for any $\widetilde{X}=\left(X_{1}, X_{2}\right), \widetilde{Y}=\left(Y_{1}, Y_{2}\right) \in T(\widetilde{M})=T\left(M_{1} \times_{f} M_{2}\right)$.

Note that for $\widetilde{J}_{+}=\frac{2 \sigma_{p, q}-p}{2} F+\frac{p}{2} I$, the projection operators are $\pi_{1}=m, \pi_{2}=l$ and for $\widetilde{J}_{-}=-\frac{2 \sigma_{p, q}-p}{2} F+\frac{p}{2} I$ we have $\pi_{1}=l, \pi_{2}=m$, where $m$ and $l$ are given by (1.6).

Remark 2.2. If we denote by $\widetilde{\nabla}$ the Levi-Civita connection on $\widetilde{M}$ with respect to $\widetilde{g}$, we obtain that $\tilde{\nabla} F=0$ [hence $\widetilde{\nabla} \widetilde{J}_{ \pm}=0$ and so $\left(\widetilde{M}=M_{1} \times_{f} M_{2}, \widetilde{g}, \widetilde{J}_{ \pm}\right)$is a locally metallic Riemannian manifold].

For the case of a product Riemannian manifold $\left(\widetilde{M}=M_{1} \times M_{2}, \widetilde{g}\right)$ with $\widetilde{g}$ given by $(2.1)$ for $f=1$ and $\widetilde{J}_{ \pm}$defined by $(2.13)$, we deduce that the Riemann curvature of $\widetilde{\nabla}$ verifies $(2.7),(2.8),(2.9),(2.10)$. 
2.2.2. Metallic Riemannian structure on $(\widetilde{M}, \widetilde{g})$ induced by two metallic structures on $M_{1}$ and $M_{2}$

For any vector field $\widetilde{X}=(X, Y) \in T\left(M_{1} \times M_{2}\right)$ we define a linear map $\widetilde{J}$ of tangent space $T\left(M_{1} \times M_{2}\right)$ into itself by:

$$
\widetilde{J} \widetilde{X}=\left(J_{1} X, J_{2} Y\right)
$$

where $J_{1}$ and $J_{2}$ are two metallic structures defined on $M_{1}$ and $M_{2}$, respectively, with $J_{i}^{2}=p J_{i}+q I, i \in\{1,2\}$ and $p, q$ non zero natural numbers. It follows that:

$$
\widetilde{J}^{2} \widetilde{X}=\widetilde{J}\left(J_{1} X, J_{2} Y\right)=\left(J_{1}^{2} X, J_{2}^{2} Y\right)=p\left(J_{1} X, J_{2} Y\right)+q(X, Y) .
$$

Also from $g_{i}\left(J_{i} X_{i}, Y_{i}\right)=g_{i}\left(X_{i}, J_{i} Y_{i}\right), i \in\{1,2\}$, we get $\widetilde{g}(\widetilde{J} \widetilde{X}, \widetilde{Y})=\widetilde{g}(\widetilde{X}, \widetilde{J} \widetilde{Y})$. Therefore, we can state the following result:

Theorem 2.3. If $\left(M_{1}, g_{1}, J_{1}\right)$ and $\left(M_{2}, g_{2}, J_{2}\right)$ are metallic Riemannian manifolds with $J_{i}^{2}=p J_{i}+q I, i \in\{1,2\}$ and $p, q$ non-zero natural numbers, then there exists a metallic Riemannian structure $\widetilde{J}$ on $(\widetilde{M}, \widetilde{g})$ given by:

$$
\widetilde{J} \widetilde{X}=\left(J_{1} X, J_{2} Y\right),
$$

for any $\widetilde{X}=(X, Y) \in T(\widetilde{M})$, where $\widetilde{M}=M_{1} \times_{f} M_{2}$ and $\widetilde{g}(\widetilde{X}, \widetilde{Y})=g_{1}\left(X_{1}, Y_{1}\right)+$ $\left(f \circ p_{1}\right)^{2} g_{2}\left(X_{2}, Y_{2}\right)$, for any $\widetilde{X}=\left(X_{1}, X_{2}\right), \widetilde{Y}=\left(Y_{1}, Y_{2}\right) \in T(\widetilde{M})=T\left(M_{1} \times_{f} M_{2}\right)$.

For the case of a product Riemannian manifold $\left(\widetilde{M}=M_{1} \times M_{2}, \widetilde{g}\right)$ with $\widetilde{g}$ given by $(2.1)$ for $f=1$ and $\widetilde{J}_{ \pm}$defined by $(2.13)$, we deduce that the Riemann curvature of $\widetilde{\nabla}$ verifies (2.7), (2.8), (2.9), (2.10).

Now we shall obtain a characterization of the metallic structure on the product of two metallic manifolds $\left(M_{1}, J_{1}\right)$ and $\left(M_{2}, J_{2}\right)$ in terms of metallic maps, that are smooth maps $\Phi: M_{1} \rightarrow M_{2}$ satisfying:

$$
T \Phi \circ J_{1}=J_{2} \circ T \Phi \text {. }
$$

In a way similar to the case of Golden manifolds $([5])$, we have:

Proposition 2.2. The metallic structure $\widetilde{J}:=\left(J_{1}, J_{2}\right)$ given by (2.16) is the only metallic structure on the product manifold $\widetilde{M}=M_{1} \times M_{2}$ such that the projections $p_{1}$ and $p_{2}$ on the two factors $M_{1}$ and $M_{2}$ are metallic maps.

A necessary and sufficient condition for the warped product of two locally metallic Riemannian manifolds to be locally metallic will be further provided: 
Theorem 2.4. Let $\left(\widetilde{M}=M_{1} \times_{f} M_{2}, \widetilde{g}, \widetilde{J}\right)$ (with $\widetilde{g}$ given by (2.1) and $\widetilde{J}$ given by (2.16)) be the warped product of the locally metallic Riemannian manifolds $\left(M_{1}, g_{1}, J_{1}\right)$ and $\left(M_{2}, g_{2}, J_{2}\right)$. Then $\left(\widetilde{M}=M_{1} \times_{f} M_{2}, \widetilde{g}, \widetilde{J}\right)$ is locally metallic if and only if:

$$
\left\{\begin{array}{l}
\left(d f^{2} \circ J_{1}\right) \otimes I=d f^{2} \otimes J_{2} \\
g_{2}\left(J_{1} \cdot, \cdot\right) \cdot \operatorname{grad}\left(f^{2}\right)=g_{2}(\cdot, \cdot) \cdot J_{1}\left(\operatorname{grad}\left(f^{2}\right)\right)
\end{array} .\right.
$$

Proof. Replacing the expression of $\widetilde{\nabla}$ from (2.4), under the assumptions ${ }^{M_{1}} \nabla J_{1}=0$ and ${ }^{M_{2}} \nabla J_{2}=0$ we obtain the conclusion.

Theorem 2.5. Let $\left(\widetilde{M}=M_{1} \times_{f} M_{2}, \widetilde{g}, \widetilde{J}\right)$ (with $\widetilde{g}$ given by (2.1) and $\widetilde{J}$ (2.16)) be the warped product of the metallic Riemannian manifolds $\left(M_{1}, g_{1}, J_{1}\right)$ and $\left(M_{2}, g_{2}, J_{2}\right)$. If $M_{1}$ and $M_{2}$ have $J_{1}$ - and $J_{2}$-invariant Ricci tensors, respectively (i.e. $Q_{M_{i}} \circ J_{i}=$ $J_{i} \circ Q_{M_{i}}, i \in\{1,2\}$ ), then $\widetilde{M}$ has $\widetilde{J}$-invariant Ricci tensor if and only if

$$
\operatorname{Hess}(f)\left(J_{1} \cdot, \cdot\right)-\operatorname{Hess}(f)\left(\cdot, J_{1} \cdot\right) \in\{0\} \times T\left(M_{2}\right) .
$$

Proof. If we denote by $S, S_{M_{1}}, S_{M_{2}}$ the Ricci curvature tensors on $\widetilde{M}, M_{1}$ and $M_{2}$ and $\widetilde{S_{M_{1}}}, \widehat{S_{M_{2}}}$ the lift on $\widetilde{M}$ of $S_{M_{1}}$ and $S_{M_{2}}$, by using Lemma 2.2 , for any $X$, $Y \in \mathcal{L}\left(M_{1}\right)$, we have:

$$
\begin{gathered}
S(\widetilde{J} X, Y)=\widetilde{S_{M_{1}}}(\widetilde{J} X, Y)-\frac{m}{f} H^{f}(\widetilde{J} X, Y)=\widetilde{S_{M_{1}}}(X, \widetilde{J} Y)-\frac{m}{f} H^{f}(\widetilde{J} X, Y)= \\
=S(X, \widetilde{J} Y)+\frac{m}{f} H^{f}(X, \widetilde{J} Y)-\frac{m}{f} H^{f}(\widetilde{J} X, Y),
\end{gathered}
$$

where $H^{f}$ is the lift on $\widetilde{M}$ of $H e s s(f)$. Also, for any $V, W \in \mathcal{L}\left(M_{2}\right)$, we obtain:

$$
\begin{aligned}
& S(\widetilde{J} V, W)=\widetilde{S_{M_{2}}}(\widetilde{J} V, W)-\left[f \Delta(f)+(m-1)|\operatorname{grad}(f)|^{2}\right] g_{2}\left(J_{2} V, W\right)= \\
& =\widetilde{S_{M_{2}}}(V, \widetilde{J} W)-\left[f \Delta(f)+(m-1)|\operatorname{grad}(f)|^{2}\right] g_{2}\left(V, J_{2} W\right)=S(V, \widetilde{J} W) .
\end{aligned}
$$

Example 2.1. Consider $M:=\left\{\left(u, \alpha_{1}, \alpha_{2}, \ldots, \alpha_{n}\right), u>0, \alpha_{i} \in\left[0, \frac{\pi}{2}\right], i \in\{1, \ldots, n\}\right\}$ and let $f: M \rightarrow \mathbb{R}^{2 n}$ be the immersion given by:

$$
f\left(u, \alpha_{1}, \ldots, \alpha_{n}\right):=\left(u \cos \alpha_{1}, u \sin \alpha_{1}, \ldots, u \cos \alpha_{n}, u \sin \alpha_{n}\right) .
$$

We can find a local orthonormal frame of the submanifold $M$ in $\mathbb{R}^{2 n}$, spanned by the vectors:

$$
Z_{0}=\sum_{i=1}^{n}\left(\cos \alpha_{i} \frac{\partial}{\partial x_{i}}+\sin \alpha_{i} \frac{\partial}{\partial y_{i}}\right), \quad Z_{i}=-u \sin \alpha_{i} \frac{\partial}{\partial x_{i}}+u \cos \alpha_{i} \frac{\partial}{\partial y_{i}}
$$

for any $i \in\{1, \ldots, n\}$. 
We remark that $\left\|Z_{0}\right\|^{2}=n,\left\|Z_{i}\right\|^{2}=u^{2}, Z_{0} \perp Z_{i}$, for any $i \in\{1, \ldots, n\}$ and $Z_{i} \perp Z_{j}$, for $i, j \in\{1, \ldots, n\}$ with $i \neq j$.

In the next considerations, we shall denote by:

$$
\left(X^{1}, Y^{1}, \ldots, X^{k}, Y^{k}, X^{k+1}, Y^{k+1}, \ldots, X^{n}, Y^{n}\right)=:\left(X^{i}, Y^{i}, X^{j}, Y^{j}\right),
$$

for any $k \in\{2, \ldots, n-1\}, i \in\{1, \ldots, k\}$ and $j \in\{k+1, \ldots, n\}$.

Let $J: \mathbb{R}^{2 n} \rightarrow \mathbb{R}^{2 n}$ be the $(1,1)$-tensor field defined by:

$$
J\left(X^{i}, Y^{i}, X^{j}, Y^{j}\right):=\left(\sigma X^{i}, \sigma Y^{i}, \bar{\sigma} X^{j}, \bar{\sigma} Y^{j}\right),
$$

for any $k \in\{2, \ldots, n-1\}, i \in\{1, \ldots, k\}$ and $j \in\{k+1, \ldots, n\}$, where $\sigma:=\sigma_{p, q}$ is the metallic number and $\bar{\sigma}=1-\sigma$. It is easy to verify that $J$ is a metallic structure on $\mathbb{R}^{2 n}$ (i.e. $J^{2}=p J+q I$ ).

Moreover, the metric $\bar{g}$, given by the scalar product $\langle\cdot, \cdot\rangle$ on $\mathbb{R}^{2 n}$, is $J$-compatible and $\left(\mathbb{R}^{2 n}, \bar{g}, J\right)$ is a metallic Riemannian manifold.

From (2.18) we get:

$$
J Z_{0}=\sigma \sum_{i=1}^{k}\left(\cos \alpha_{i} \frac{\partial}{\partial x_{i}}+\sin \alpha_{i} \frac{\partial}{\partial y_{i}}\right)+\bar{\sigma} \sum_{j=k+1}^{n}\left(\cos \alpha_{j} \frac{\partial}{\partial x_{j}}+\sin \alpha_{j} \frac{\partial}{\partial y_{j}}\right)
$$

and, for any $k \in\{2, \ldots, n-1\}, i \in\{1, \ldots, k\}$ and $j \in\{k+1, \ldots, n\}$ we get:

$$
J Z_{i}=\sigma Z_{i}, \quad J Z_{j}=\bar{\sigma} Z_{j} .
$$

We can verify that $J Z_{0}$ is orthogonal to $\operatorname{span}\left\{Z_{1}, \ldots, Z_{n}\right\}$ and

$$
\cos \left({\widehat{J Z_{0}, Z_{0}}}=\frac{k \sigma+(n-k) \bar{\sigma}}{\sqrt{n\left(k \sigma^{2}+(n-k) \bar{\sigma}^{2}\right)}} .\right.
$$

Consider the manifolds $M_{1}$ and $M_{2}$ with $T M_{1}=\operatorname{span}\left\{Z_{0}\right\}$ and $T M_{2}=\operatorname{span}\left\{Z_{1}, \ldots, Z_{n}\right\}$. Then $M:=M_{1} \times_{u} M_{2}$ with the Riemannian metric tensor $g=n d u^{2}+u^{2} \sum_{i=1}^{n} d \alpha_{i}^{2}$ is a warped product (semi-slant) submanifold of the metallic Riemannian manifold $\left(\mathbb{R}^{2 n},\langle\cdot, \cdot\rangle, J\right)$.

Acknowledgements. The authors thank the referee for his/her valuable suggestions that definitely improved the paper.

\section{REF E R E N C E S}

1. M. Atceken: Warped Product Semi-Invariant Submanifolds in locally decomposable Riemannian manifolds. Hacet. J. Math. Stat. 40, no. 3, (2011), 401-407.

2. M. Atceken and S. Keles: On the product Riemannian manifolds. Differ. Geom. Dyn. Syst. 5, no.1, (2003), 1-8.

3. Y. B. BAIK: A certain polynomial structure. J. Korean Math. Soc. 16(80), no. 2, (1979), 167-175.

4. R. L. Bishop and B. O'Neill: Manifolds of negative curvature. Trans. Amer. Math. Soc. 145 (1969), 1-49. 
5. A. M. Blaga and C. E. Hretcanu: Golden warped product Riemannian manifolds. accepted in Libertas Mathematica.

6. M. Crasmareanu and C. E. Hretcanu: Golden differential geometry. Chaos Solitons Fractals 38(5) (2008), 1229-1238.

7. M. Crasmareanu, C. E. Hretcanu and M. I. Munteanu: Golden- and product-shaped hypersurfaces in real space forms. Int. J. Geom. Methods Mod. Phys. 10(4) (2013), paper 1320006, 9 pp.

8. S. I. GoldBerg and K. YANO: Polynomial structures on manifolds. Kodai Math. Sem. Rep. 22 (1970), 199-218.

9. S. I. Goldberg and N. C. Petridis: Differentiable solutions of algebraic equations on manifolds. Kodai Math. Sem. Rep. 25 (1973), 111-128.

10. A. N. HATzinikitas: A note on doubly warped product spaces, arXiv:1403.0204v1.2014, Physics.

11. C. E. Hretcanu and M. C. Crasmareanu: On some invariant submanifolds in Riemannian manifold with Golden Structure. An. Ştiinţ. Univ. Al. I. Cuza Iaşi. Mat. (N.S.) 53 (2007), Suppl., 199-211.

12. C. E. Hretcanu and M. C. Crasmareanu: Applications of the Golden Ratio on Riemannian Manifolds. Turkish J. Math. 33(2) (2009), 179-191.

13. C. E. Hretcanu and M. C. Crasmareanu: Metallic structures on Riemannian manifolds. Rev. Un. Mat. Argentina 54(2) (2013), 15-27.

14. W. J. LU: $f$-Harmonic maps of doubly warped product manifolds. Appl. Math. J. Chinese Univ. 28 (2013), 240-252.

Adara M. Blaga

West University of Timişoara

Timişoara, 300223, Romania

adarablaga@yahoo.com

Cristina E. Hreţcanu

Stefan cel Mare University of Suceava

Suceava, 720229, Romania

criselenab@yahoo.com 